\title{
Volumetric Analysis of Brain Tumor Magnetic Resonance Image
}

\author{
$1^{\text {st }}$ Hapsari Peni Agustin \\ Tjahyaningtijas \\ Department of Electrical Engineering, \\ Institut Teknologi Sepuluh Nopember, \\ Surabaya, Indonesia \\ Department of Electrical Engineering \\ Universitas Negeri Surabaya \\ Surabaya, Indonesia \\ e-mail: hapsari.17071@mhs.its.ac.id
}

\author{
$2^{\text {nd }}$ Hanik Badriyah Hidayati \\ Neurology Department, \\ Faculty Medicine \\ Airlangga University \\ Surabaya, Indonesia \\ hanikhidayati@fk.unair.ac.id \\ $3^{\text {rd }}$ Adri Gabriel Sooai \\ Dept. of Computer Science \\ Universitas Katolik Widya Mandira \\ Kupang, Indonesia \\ adrigabriel@ieee.org
}

\author{
$4^{\text {th }}$ I Ketut Eddy Purnama \\ Dept. of Electrical Engineering \\ Dept. of Computer Engineering \\ Institut Teknologi Sepuluh Nopember \\ Surabaya, Indonesia \\ ketut@ee.its.ac.id \\ $5^{\text {th }}$ Mauridhi Hery Purnomo \\ Dept. of Electrical Engineering \\ Dept. of Computer Engineering \\ Institut Teknologi Sepuluh Nopember \\ Surabaya, Indonesia \\ hery@ee.its.ac.id
}

\begin{abstract}
Volumetric analysis of brain tumors is a decisive thing in the detection of brain tumors to determine the patient's lifetime followed by action to the patient. A few studies had been shown explicitly quantified the brain tumor volume while the analysis of brain tumor volumetric by expert limited with the huge data of brain tumor patient MRI. Thorough the importance of brain tumor analysis in clinical used, the purpose of this research is to evaluate the similarity of a semi-automatic segmentation tool for brain tumor image analysis. The agreement was compared by using differences of means with $95 \%$ limits of agreement (LoA). Brain tumor segmentation was obtained by using Fast Marching and Grow Cut segmentation methods. Preoperative MRI images of 20 T2 MRI of low-grade glioma patients from The Cancer Imaging Archive (TCIA) database were used to analyze brain tumor volume. The volume obtained from the two segmentation methods is based on the similarity between the two using the intra-method agreement between two segmentation methods with a $95 \%$ limit of agreement (LOA) value and difference volume average of $920 \mathrm{~mm}^{3}$ or $0.92 \mathrm{~mL}$. Its shown that both methods had the same performance.
\end{abstract}

Keywords-Fast Marching, Grow Cut, Limits of Agreement, Difference Volume Average.

\section{INTRODUCTION}

Brain tumors are abnormal brain cancer tissue growths that are uncontrolled. Tumors of the brain may be benign and malignant. Benign tumors have a homogeneous composition and have cancer cells that are inactive. Malignant tumors are structurally heterogeneous and contain active cancer cells that spread to all areas. Examples of low-level tumors are gliomas and meningiomas, both of which are categorized as benign tumors, whereas glioblastoma and astrocytomas are categorized as high-level tumors. [1].

The scoring system scale is used from class I to class IV, according to the world health organization. These values classify tumor kinds that are benign and malignant. Class I and II are tumors of low grade, whereas grades III and IV are tumors of high grade. Tumors of the brain can influence people in any era. The impact may not be the same for each individual. The diagnosis of tumor fields in the brain is a difficult task due to the complex structure of the human brain. Malignant kinds of Class III and IV tumors are growing quickly so that these cells may impact healthy brain cells, spreading to other areas of the brain or spinal cord, becoming more hazardous and untreated. The number of people with brain cancer in Indonesia cannot be ascertained, but based on statistical data released by HPV and Cancer (2016), brain cancer cases in Indonesia in 2012 were 1.9 per 100,000 population, while the brain cancer mortality rate was 1.3 per 100,000 inhabitants. From these data, it was shown that the rate of people with brain cancer in Indonesia is high [2]. By paying attention to this, the identification of brain tumor cases from the beginning is very necessary to improve the quality of life of patients. In improving the quality of life of patients, the management of brain tumor tumors, which need to be considered are age, general health, tumor size, tumor location and type of tumor.

One effort that can be done is to help the process of early diagnosis of brain cancer. The process of early diagnosis of brain cancer is an important process in indicating the presence of brain cancer in humans. If brain cancer can be known early, further treatment can be done for patients. According to the Indonesian Ministry of Health, there are several steps in the process of diagnosing brain cancer, namely: (1) physical examination, is an examination of complaints that arise in patients such as vomiting, seizures, headaches and others; (2) neuro-ophthalmologic examination, which is a useful examination to explain the suitability of clinical disorders with functional brain cancer; (3) laboratory examination, namely examination of the general condition of the patient, such as liver function, kidney, blood sugar, hepatitis $\mathrm{B}$ and $\mathrm{C}$, LDH, hemostasis, and electrolytes; and (4) radiological examination, which is a useful examination to determine the location and size of cancer that grows in the brain. The standard radiological examination consists of computerized tomography (CT) scan and magnetic resonance images (MRI). The process of reading the results of the MRI is done manually, namely by observing and analyzing MRI images carried out by the radiology department, then the results of the analysis are consulted by the doctor. In reading the results MRI can only know normal or abnormal.

The development of brain tumors is analyzed through a measure of brain tumor volume, especially in cases of malignant brain tumors because it requires surgery, while in the case of benign tumors that do not require surgery, the size of the brain tumor is needed for the prognosis of pediatric patients [3]. Several studies have evaluated tumor size in one dimension, two dimensions and volumetric. The 
study of tumor size focuses more on malignant brain tumors in adult patients while studies of benign tumor volume are more focused on pediatric patients. Comparative studies of malignant brain tumors in children were written by Warren et al [4].

Brain tumor segmentation method was divided into three namely manual segmentation, semi-automatic segmentation, and automatic segmentation. For semi-automatic segmentation methods, human intervention is still needed in extracting the volume from brain tumors. Human interaction is needed to provide input for several parameters and is responsible for analyzing visual results from computer software [5].

In this study, brain tumor volume was obtained using the help of software to overcome human bias and can handle large data. Volumetric brain tumor analysis obtained from MRI of low-grade glioma patients through the Grow Cut and Fast Marching semi-automatic segmentation method using the help of 3D Slicer. Determination of brain tumor volume needs to be accepted as an agreement limit (LOA) between two techniques of segmentation. Validation of volume tumors is a challenging process and requires a long process and requires acceptable limits of agreement (LOA) between two methods. In this paper, the use of Bland-Altman analysis is an appropriate way to perform this comparison and presents quantified measures to decide whether the new method is acceptable or not.

\section{RELATED PRIOR WORK}

\section{A. Segmentation Method}

Multislice MRI brain tumor segmentation is very challenging because of the nature of the tumor in terms of size, appearance, and location. Image analysis uses a semiautomatic or automatic segmentation method to illustrate images taken. The number of clinical data becomes its own difficulty to manually determine and segment data in a timely manner. Medical image analysis is divided into image quality improvement, segmentation, visualization, quantization and modeling[6]. Segmentation and modeling are the most important and challenging in the study of brain tumors. Based on the importance of early diagnosis of brain cancer, many researchers have conducted research into the diagnosis of brain cancer. For multimodal brain tumors, there are two types of segmentation methods, namely semiautomatic and automatic. Semi-automatic segmentation methods are presented in[7]. The method has the advantage of being faster segmentation time, manual initialization sketching approximately ROI that covers the tumor. Regional-level set, edge, and active contour approaches are image analysis techniques combined in algorithms. In the automatic segmentation method, tumor contours are obtained without human intervention. When the tumor type does not match the segmentation model as learned from the training dataset, the results of the segmentation may be wrong. Both automatic segmentation methods and semi-automatic segmentation methods have advantages and disadvantages. The automatic segmentation method does not require human intervention in determining the area of the tumor and requires longer computational time. While the semiautomatic segmentation method still requires human intervention in determining the area of the tumor. Human interaction is needed periodically to ensure the accuracy results obtained even though in reality the semi-automatic segmentation method only requires human interaction when initial initialization. Nowadays a faster technique is needed to reduce time consumption in initial initialization. The faster semiautomatic segmentation method with easier initialization process and efficient modification has been developed by Menze [7]. Users only need to do manual sketches to determine ROI in the area around the tumor. The algorithm used is by combining edge base active contour with regionbased active contour. In this paper, we focus on the segmentation method using the help of 3D Slicer software to get the volume of a brain tumor based on active countour and region growing method.

\section{B. Volumetric Analysis}

In increasing the life expectancy of patients with brain tumors, the volumetric analysis is needed for tumor grading[8]. The method of analyzing the tumor area begins with the 1D approach. In this 1D measurement, the longest diameter of the tumor area was determined for each slice of the axial, sagittal or coronal MRI plane. For the 2D approach, the tumor area is obtained by the largest diameter multiplication perpendicular to the plane. Volume analysis in $3 \mathrm{D}$ is obtained by multiplying the $2 \mathrm{D}$ area with the longest vertical diameter perpendicular to the $2 \mathrm{D}$ plane [9]. This study describes the application of qualitative image analysis techniques to obtain an estimate of brain tumor volume. The application of the stereological method for MRI analysis as pioneered by the Magnetic Resonance and Image Analysis Research Centre (MARIARC) defines the statistical analysis of geometric parameters of the image sample. The stereological method applied consists of two stages. The first is two-dimensional (2D) and the next stage is the threedimensional (3D) stages

Because of the importance of analyzing tumor volume for patient prognosis, many researchers have carried out a volumetric analysis of these brain tumors. Tumor volumetric analysis was done by comparing tumor volume measurements at the beginning of detection to the next scan with the help of VITREA 2 computer software with the MRI T1 modality [9]. Brain tumor volume has been obtained using MedVision 1.41 computer software. In 49 cases of malignant brain tumors by analyzing ROI to get the volume. The average intra-observer error using this program is around $1.8 \%$ [8]. MRI T1 of 20 glioblastoma patients was segmented twice using the Brain Voyager and ITK software with two researchers. Intra rater and inter-rater between the two software are compared using limits of agreement (LoA), dice score coefficient (DSC), Hausdorff distance (HD) and time consumption. Between software, agreement reaches 95\% LoA[10].

\section{Statistical Consideration}

Correlation is a statistical method that can show the relationship between a variable. There are several correlation methods including the Pearson correlation. The results of the two data correlation are expressed with correlation coefficients or $r$. Correlation coefficients are calculated as a comparison between variables with standard deviation multiplication. The value of $r$ is between -1 and 1 . The closeness relationship between variables is indicated by the correlation coefficient value of +1 or -1 . Data correlation calculations are closely related to linear regression calculations. Linear regression is calculated only if there is a 
correlation and the correlation coefficient is interpreted as a significant $\mathrm{P}$-value. The significance of $\mathrm{P}$ and regression can be calculated for all comparative methods. Linear correlation and regression state the relationship between two data sets.

Variations in properties used in a selected set of samples must correlate well to measure the same variables. Both methods are expected to be able to measure these variables. In the case of a method comparison, this means that the sample must cover a wide range of concentrations. The high correlation for each of the two methods designed to measure the same property.

\section{Proposed METHOD}

This study combines the use of developed algorithms and clinical applications using 3D slicer devices. The input image is DICOM image. In the preprocessing step, the bias correction applied to reduce the MRI noise. The fast marching and grow cut was used for volume segmentation. Both types of segmentation are region-based segmentation. The segmented volume then analyses using Bland Altman method to form the correlation and similarity between them. The proposed diagram is shown in Fig. 1.

\section{A. N4ITK Bias Fields Correction}

A bias field can be produced because of an undesirable low-frequency signal that damages the MRI image [11], [12]. The inhomogeneity magnetic field of the MRI machine will cause the bias field. The bias field blurs the image and magnifies reducing the frequency of images from the image such as edges and contour and changing the pixel value of the image so the same image has a different gray level distribution throughout the image. Low-level variations will not have a major impact on clinical diagnosis. However, the performance of image algorithms such as segmentation and algorithms decreased based on the approval of the spatial invariance of the required images. Preprocessing steps are needed to correct the bias effect before segmentation and classification process.

\section{B. Fast Marching}

The reason for building up the fast marching method is the active contour segmentation. This technique is segmenting areas that have similar intensities. Initialization is finished by segmenting the area to get the volume structure. The consequent volume can be adjusted cleverly by glancing through the progression of the name frame Fast Marching. Segmentation procedure of this module contains the two phases: Initialization and alteration of segmentation result. The phase of initialization is input volume, input seeds, directed segmented volume, and yield mark esteem. While adjustment division result stages are segmented volume determination extends, output segmented volume and intelligent volume rendering of segmentation results [13].

\section{Grow Cut}

Grow Cut segmentation method work based on region growing algorithm. The initial seed pixel was used as an algorithm input to compute a region of interest automatically. The algorithm labeled the pixels iteratively using the initial input before. Next, ROI was labeled and no pixel can change it's label anymore [14].

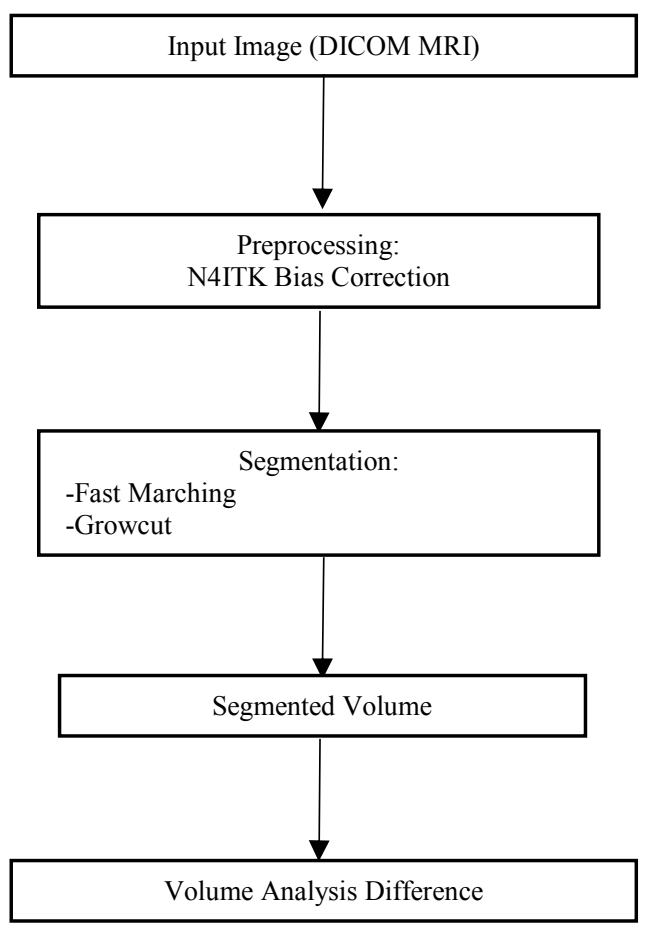

Fig.1. Blog diagram of Proposed Method

Region Growing is an approach method for image segmentation by starting with a few pixels (seeds) which represent different image regions and grow to form a wider area in the image[15]. To use this method, rules are needed which explain the growth mechanism and the rules of homogeneity of each area after growing. But in this study, the growing region is not started from seeds but from the initial initialization of the region obtained from binary segmentation. By using the initial initialization region the region growing process becomes faster in time complexity. The first step is to determine the initialization. Then the region will develop into an actual region by detecting whether the outer pixels of the region have membership criteria from the region. This algorithm is commonly used for object segmentation in various multi-resolution and multispectral image characteristics that are influenced by several factors such as image layer weight, scale parameter, shape and color, and smoothness and compactness.

\section{The analysis of differences: the Bland and Altman method}

In this research, the Bland-Altman plot (B\& $\mathrm{A})$ is used to describe the relationship between two quantitative methods by applying a method to measure the agreement between two methods with a limit of agreement (LoA) [15]. The statistical limit used is the mean and standard deviation (s) between two methods. The graphical approach is used to determine the assumption of normality difference.

The Bland-Altman plot uses a plot distribution with the $\mathrm{Y}$-axis the difference between two data sets $\left(\mathrm{S}_{1}-\mathrm{S}_{2}\right)$ and the $\mathrm{X}$-axis expressing the average of $\left(\left(\mathrm{S}_{1}+\mathrm{S}_{2}\right) / 2\right)$. Furthermore, a graph is made between the differences between the two methods of the average. Data points must be $95 \%$ within $\pm 2 \mathrm{~s}$ of the average difference according to the $\mathrm{B} \& \mathrm{~A}$ recommendation. The Bland-Altman Plot follow the equation below 


$$
\begin{aligned}
& S(x, y)=\left(\frac{S_{1}+S_{2}}{2}, S_{1}-S_{2}\right) \\
& S(x, y)=\left(\frac{\log _{2} S_{1}+\log _{2} S_{2}}{2}, \log _{2} S_{1}-\log _{2} S_{2}\right)
\end{aligned}
$$

\section{EXPERIMENT AND RESULT}

The experiment was carried out using TCIA MRI T2 image data set of low-grade glioma tumors consisting of 20 patients. Each patient's MRI image consisted of 20-40 images with $0.9375 \times 0.9375$ pixel spacing and slice thickness of $3 \mathrm{~mm}$.

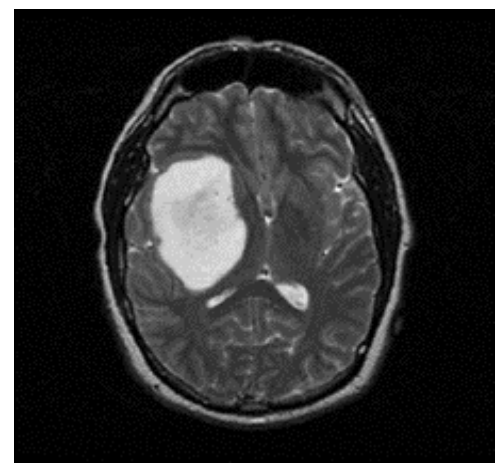

Fig. 1. LGG MRI image

Dataset files used in the form of DICOM images are processed using 3D Slicer to obtain brain tumor volume using Fast Marching and Grow Cut segmentation methods. The segmentation results of the methods are shown in the figure below.

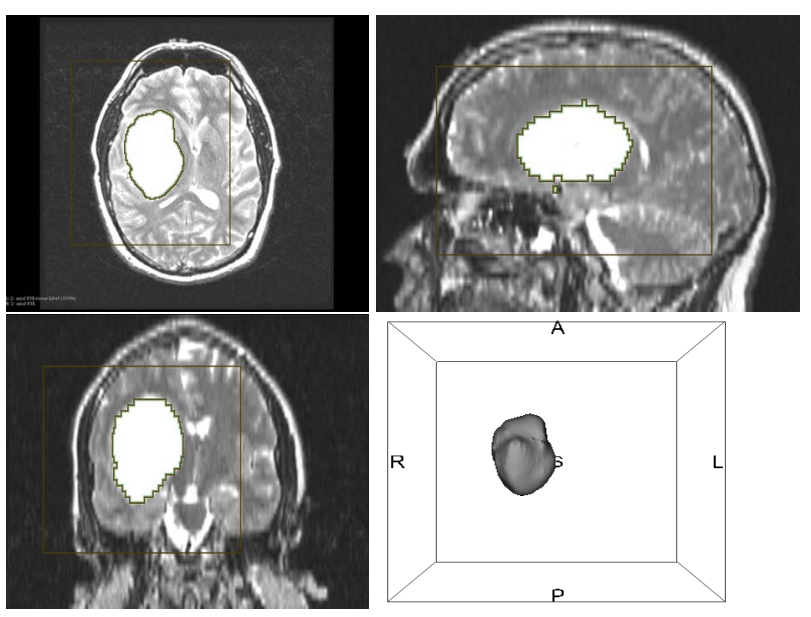

Fig. 2. Grow Cut segmentation result and volume rendering

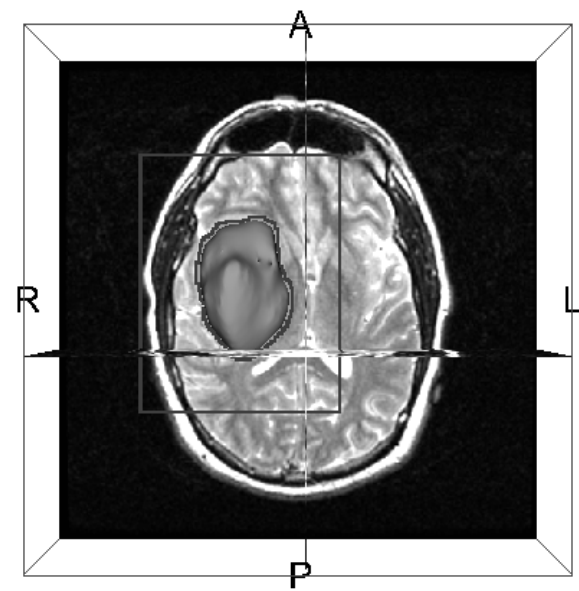

Fig. 3. Model of the resulting tumor segmentation using Grow Cut method
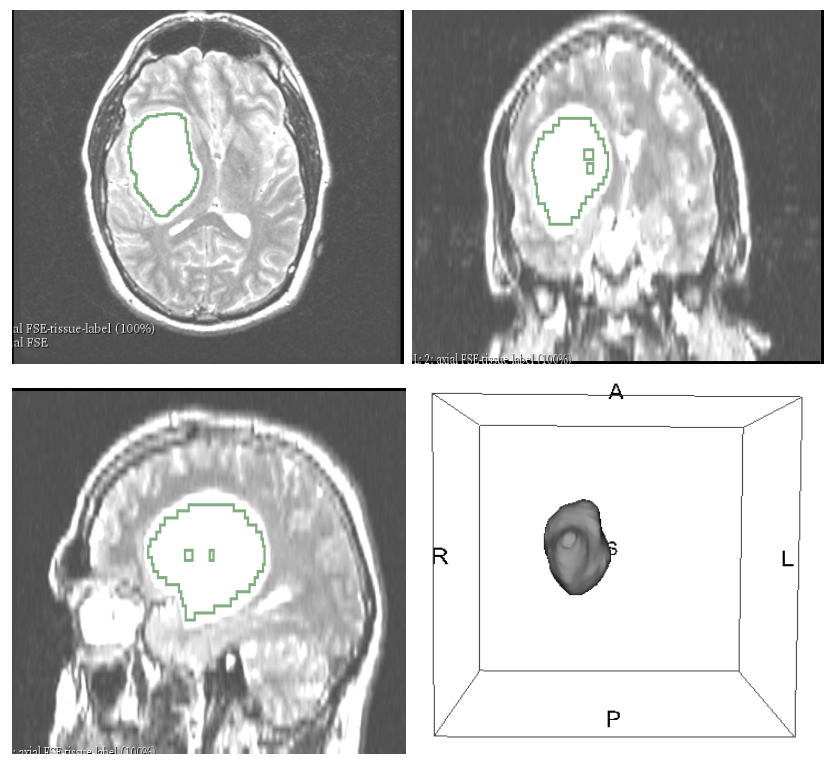

Fig. 4. Fast Marching segmentation result and volume rendering

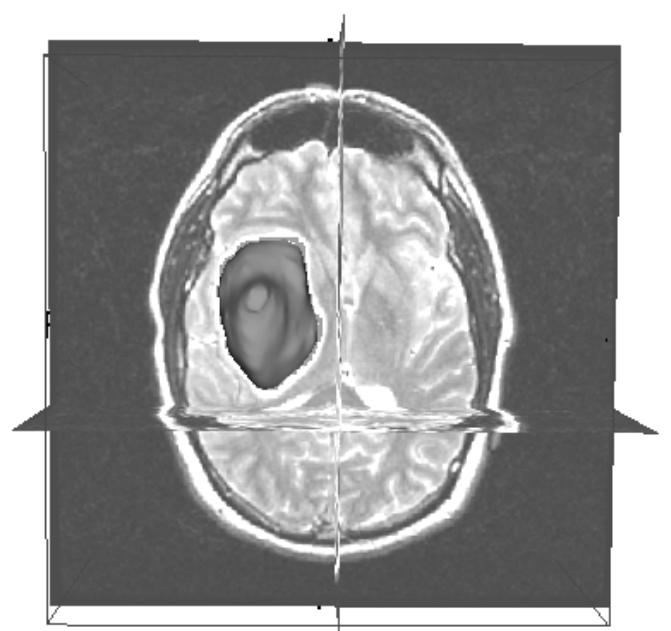

Fig. 5. Model of the resulting tumor segmentation using Fast Marching Method. 
The volume of brain tumor measured was listed below:

TABLE 1. BRAIN TUMOR VOLUME EXTRACTED USING 3D SLICER

\begin{tabular}{|c|c|c|c|}
\hline \multirow{2}{*}{ Case } & \multicolumn{3}{|c|}{ Brain Tumor Volume (mm3) } \\
\hline & Fast Marching & Grow Cut & Ground Truth \\
\hline 1 & 13181,16 & 15023,53 & 15896,88 \\
\hline 2 & 56420,96 & 40373,04 & 46046,73 \\
\hline 3 & 63898,86 & 61845,74 & 59912,93 \\
\hline 4 & 7648,30 & 12036,80 & 7349,71 \\
\hline 5 & 9141,46 & 9575,67 & 8435,82 \\
\hline 6 & 23077,30 & 23721,13 & 23938,80 \\
\hline 7 & 13144,55 & 12037,80 & 15292,48 \\
\hline 8 & 22186,02 & 23715,00 & 27873,34 \\
\hline 9 & 63780,34 & 66358,58 & 64289,20 \\
\hline 10 & 15597,23 & 18617,09 & 19902,35 \\
\hline 11 & 5611,11 & 6258,05 & 5499,38 \\
\hline 12 & 21719,53 & 31598,83 & 35064,27 \\
\hline 13 & 60189,89 & 59715,32 & 66579,88 \\
\hline 14 & 12130,19 & 10858,69 & 15269,06 \\
\hline 15 & 20603,98 & 20217,50 & 17594,49 \\
\hline 16 & 85669,75 & 86431,23 & 80454,74 \\
\hline 17 & 28964,83 & 26876,71 & 30167,69 \\
\hline 18 & 25185,02 & 26586,57 & 29745,43 \\
\hline 19 & 26099,00 & 29315,21 & 24475,26 \\
\hline 20 & 14107,49 & 12601,61 & 14538,83 \\
\hline
\end{tabular}

The volume difference between segmentation relative to tumor volume is measured by calculating the volume difference between segmentation methods. The difference was analyzed using B\&A plot.

TABLE 2. BRAIN TUMOR VOLUME ANALYSIS

\begin{tabular}{|l|c|c|}
\hline Volume Analysis & $\begin{array}{c}\text { Difference volume } \\
\left(\mathrm{mm}^{3}\right)\end{array}$ & $\begin{array}{c}\text { Difference Mean } \\
\left(\mathrm{mm}^{3}\right)\end{array}$ \\
\hline Bias & $-270,36$ & 29553,03 \\
\hline Standart Deviation & 4715,05 & 22547,85 \\
\hline Lower LoA & $-9511,85$ & $-14640,76$ \\
\hline Higher LoA & 8971,13 & 73746,81 \\
\hline
\end{tabular}

The results of the experiment are shown in Table 1 and Table 2. The tumor volume comparison is presented in Table 1 , while the ground truth volume calculated using manual calculation under neurologist supervission. Table 2, B \& A plot and agreement of evaluation are created. A series of volume readings, acquired by a technique called Fast Marching segmentation, are displayed in the first column. The second column indicates the measurements for Grow Cut segmentation, the distinct technique. Therefore, the paired information is shown in each row. Fig. 6 Displays the line of regression between the two methods; the coefficient of correlation between the two methods is $r=0.9792(95 \%$ confidence interval, $\mathrm{CI}=0.991-0.998, \mathrm{P}<0.001)$, and the equation of regression is $y=2060.6+0.9391 x$; this could be considered as a very good agreement.

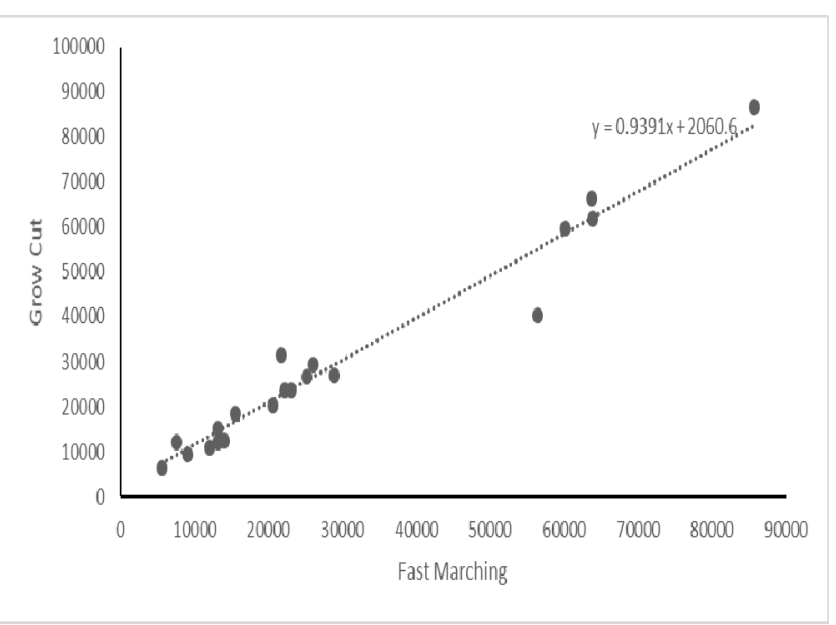

Fig. 6. Linear Regression of Fast Marching and Grow Cut

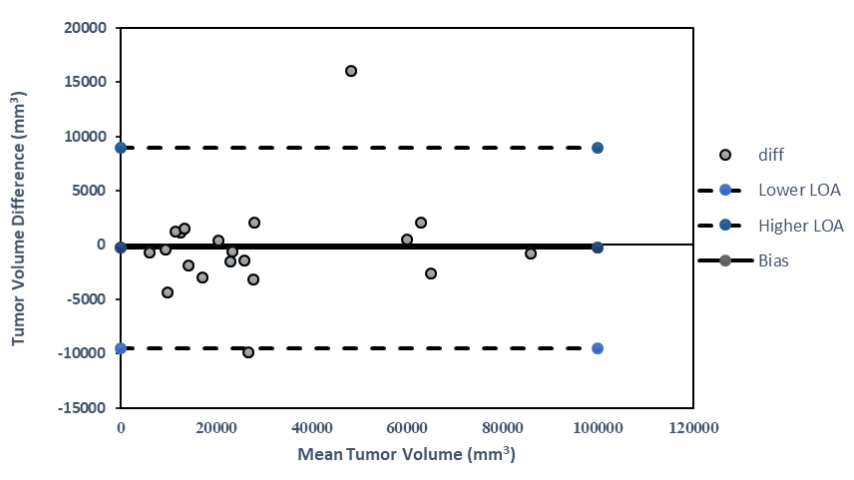

Fig. 7. . LoA Bland\&Altman plots

The inter-rater agreement was calculated as a tumor volume difference value with $95 \%$ of the LoA between the first method of segmentation and the second method segmentation., as described by Bland and Altman [16]. Bland-Altman plots [15] with 95\% LoA were created from equation (1) and (2).

The difference in tumor volumes for a combined mean of segmentation between the two methods plotted against mean tumor volume of each segmentation (Fig.7). The whole line represents mean difference, stapled lines represent 95\% limits of agreement. Fig. 7 of Bland-Altman Plot showed that the $95 \%$ confidence interval for the mean is [18588.27, 40247.42], while the $95 \%$ confidence interval for the levels of agreement is [-28337.31, 26600.73].

\section{DISCUSSION}

This study discusses the agreement between two segmentation methods, Fast Marching and Growcut for LGG segmentation. The calculation results indicate the existence of a high agreement and correlation between the two. The agreement obtained is in accordance with $95 \%$ of the LoA on the B \& A plot while the correlation value is stated with a high correlation coefficient. This shows that the two methods have a close relationship only in the Fast Marching method 
still requires more time in the segmentation process compared to Grow Cut.

The mean difference value is compared with the volume difference value by applying the B \& A plot method with 95\% LoA. Two data sets show the same performance. Both data sets show a high correlation with each other. The average segmented volume for the Fast Marching method is higher than the Grow Cut method. The Grow Cut method gives a sharper picture of the segmented edge of the tumor so that the visualization of the tumor area is clearer. Because visualization of the human eye tends to see differences, in contrast, the Grow Cut method is more helpful in measuring semi-automatic brain tumors.

Analysis of the volume mean difference of the two data sets shows that the smaller difference value, the better the volume size, while the greater the difference in the mean difference value, the further the tumor volume will be.

The two methods of segmentation may also be clinically applicable. Pre-operative 3D segmentation may enhance the visualization of the surgeon during both planning and intraoperative use of neuro-navigation. Volume segmentation may also be useful in individual patients to plan radiotherapy or evaluate treatment effects.

Limitations of this study for everyday clinical work are the methods used. The method used is still semi-automatic. It is necessary to develop a software engine that is able to automatically segment and visualize the volume of brain tumors appropriately Visualizing the correct quantity of preoperative brain tumors will improve the specialist's representation both during planning and when using neuronavigation intraoperatively. It is essential that state-of - theart algorithms are introduced in user-friendly graphical user interfaces that are available to clinicians and technicians alike in order for segmentation to become part of everyday exercise.

There was no standardization of the scan parameters acquired. It is unlikely to add to bias by evaluating variations in means, although the accuracy of volume estimates may differ with image quality. However, one may claim that with distinct scanners and scanning parameters this closer resembles the daily workflow.

\section{CONCLUSION}

In this reseacrh, we discovered the segmentation technique, Fast Marching and Grow Cut on 3D Slicer have similar effectiveness in terms of high contract volume intrarater, inter-rater and inter-software and spatial overlap for LGG segmentation. Fast Marching method was significantly longer than in the Grow Cut segmentation, but the smoothness of the workflow could be better than other.

\section{ACKNOWLEDGMENT}

The writers would like to thank the Indonesian government for the BPPDN scholarship for their assistance through the scholarship program for Indonesian education.

\section{REFERENCES}

N. B. Bahadure, A. K. Ray, and H. P. Thethi, "Image Analysis for MRI Based Brain Tumor Detection and Feature Extraction Using Biologically Inspired BWT and SVM,” Int. J. Biomed.
Imaging, vol. 2017, pp. 1-12, Mar. 2017.

[2] Komite Penanggulangan Kanker Nasional, Pedoman Nasional Pelayanan Kedokteran Tumor Otak. 2017.

[3] F. D'Arco, P. O’Hare, F. Dashti, A. Lassaletta, T. Loka, U. Tabori, G. Talenti, S. Thust, G. Messalli, P. Hales, E. Bouffet, and S. Laughlin, "Volumetric assessment of tumor size changes in pediatric low-grade gliomas: feasibility and comparison with linear measurements," Neuroradiology, vol. 60, no. 4, pp. 427436, Apr. 2018.

[4] K. E. Warren, N. Patronas, A. A. Aikin, P. S. Albert, and F. M. Balis, "Comparison of one-, two-, and three-dimensional measurements of childhood brain tumors.," J. Natl. Cancer Inst., vol. 93, no. 18, pp. 1401-5, Sep. 2001.

[5] H. Peni Agustin Tjahyaningtijas, "Brain Tumor Image Segmentation in MRI Image," IOP Conf. Ser. Mater. Sci. Eng., vol. 336, no. 1, p. 012012, Apr. 2018.

[6] I. N. (Isaac N. . Bankman, Handbook of medical image processing and analysis. Elsevier/Academic Press, 2009.

[7] B. Menze, M. Reyes, A. Jakab, E. Gerstner, J. Kirby, and K. Farahani, "Proceedings of the MICCAI Challenge on Multimodal Brain Tumor Image Segmentation (BRATS) 2013," MICCAI Chall. Multimodal Brain Tumor Image Segmentation, vol. 57, 2013.

[8] W. M. Shi, D. M. Wildrick, and R. Sawaya, "Volumetric measurement of brain tumors from MR imaging.," $J$. Neurooncol., vol. 37, no. 1, pp. 87-93, Mar. 1998.

[9] G. D. Shah, S. Kesari, R. Xu, T. T. Batchelor, A. M. O’Neill, F. H. Hochberg, B. Levy, J. Bradshaw, and P. Y. Wen, "Comparison of linear and volumetric criteria in assessing tumor response in adult high-grade gliomas1," Neuro. Oncol., vol. 8, no. 1, pp. 38-46, Jan. 2006.

[10] E. H. Fyllingen, A. L. Stensjøen, E. M. Berntsen, O. Solheim, and I. Reinertsen, "Glioblastoma Segmentation: Comparison of Three Different Software Packages," PLoS One, vol. 11, no. 10, p. e0164891, Oct. 2016.

[11] N. J. Tustison, B. B. Avants, P. A. Cook, Y. Yuanjie Zheng, A. Egan, P. A. Yushkevich, and J. C. Gee, "N4ITK: Improved N3 Bias Correction," IEEE Trans. Med. Imaging, vol. 29, no. 6, pp. 1310-1320, Jun. 2010.

[12] I. Despotović, B. Goossens, and W. Philips, "MRI segmentation of the human brain: challenges, methods, and applications.," Comput. Math. Methods Med., vol. 2015, p. 450341, 2015.

[13] Xinyang Liu, K. Tuncali, W. M. Wells, P. R. Morrison, and G. P. Zientara, "Fully automatic 3D segmentation of iceball for imageguided cryoablation," in 2012 Annual International Conference of the IEEE Engineering in Medicine and Biology Society, 2012, pp. 2327-2330.

[14] Y. Boykov and G. Funka-Lea, "Graph Cuts and Efficient N-D Image Segmentation," Int. J. Comput. Vis., vol. 70, no. 2, pp. 109-131, Nov. 2006.

[15] J. M. Bland and D. G. Altman, "Measuring agreement in method comparison studies," Stat. Methods Med. Res., vol. 8, no. 2, pp. 135-160, Apr. 1999.

[16] J. M. Bland and D. G. Altman, "Statistical methods for assessing agreement between two methods of clinical measurement.," Lancet (London, England), vol. 1, no. 8476, pp. 307-10, Feb. 1986. 